\title{
Robust Adaptive Control of Nonlinear Systems with Unknown State Delay
}

\author{
Alper Bayrak, Enver Tatlicioglu, Baris Bidikli, and Erkan Zergeroglu
}

\begin{abstract}
In this work, we propose a new robust adaptive controller for a class of multi-input multi-output nonlinear systems subject to uncertain state delay. The proposed method is proven to yield semi-global asymptotic tracking despite the presence of additive input and output disturbances and parametric uncertainty in the system dynamics. An adaptive desired system compensation in conjunction with a continuous nonlinear integral feedback component is utilized in the design of the controller and Lyapunov-based techniques, are used to prove that the tracking error is asymptotically driven to zero. Numerical simulation results are presented to demonstrate the effectiveness of the proposed method.
\end{abstract}

\section{INTRODUCTION}

Adaptive control of nonlinear systems subject to structured and unstructured uncertainties with unknown/uncertain state delay has received noteworthy attention over the last several years [1]. As a result, various robust adaptive controllers, based on different model assumptions and stability results, have been proposed. To name a few, in [2], Zhang and Ge proposed an adaptive neural controller for a class of uncertain multi-input multi-output (MIMO) nonlinear systems in a triangular control structure with unknown state delay. The design was based on the principle of sliding mode control and the unknown time-varying delays were compensated by the use of appropriate Lyapunov-Krasovskii functionals. In [3], [4], [5], researchers have proposed adaptive neural network based tracking controllers for nonlinear time delay systems. A discontinuous adaptive controller for a class of nonlinear systems with unknown time delay was proposed in [6]. In [7], Zheng et al. designed an adaptive robust controller for uncertain linear systems with multiple state delays. Hua et al., in [8], studied adaptive backstepping control of a class of nonlinear time delay systems with triangular structure. Tong and Sheng [9] proposed an adaptive fuzzy backstepping controller for a class of nonlinear systems with unknown time delay and unmeasured states. One drawback of the studies in [2]-[9], however, were the uniform ultimate bounded stability results. Furthermore, there are singularity issues in the controllers of [4] and [6]. In [10], Wu designed an adaptive robust controller for uncertain nonlinear systems subject to nonlinear delayed state perturbations. However, while the perturbations were assumed to be unknown with a bounded

A. Bayrak and E. Tatlicioglu are with the Department of Electrical \& Electronics Engineering, Izmir Institute of Technology, Izmir, 35430 Turkey (Phone: +90 (232) 7506536; Fax: +90 (232) 7506599; E-mail: [alperbayrak,envertatlicioglu] @iyte.edu.tr).

B. Bidikli is with the Department of Electrical \& Electronics Engineering, Celal Bayar University, Manisa, Turkey (E-mail: barisbidikli@cbu.edu.tr).

E. Zergeroglu is with the Department of Computer Engineering, Gebze Institute of Technology, 41400, Gebze, Kocaeli, Turkey (Email: ezerger@bilmuh.gyte.edu.tr). magnitude, the rest of the system dynamics was assumed to be known exactly. In [11], Mirkin and Gutman proposed an output feedback model reference adaptive control scheme for a class of MIMO linear dynamic systems with unknown state delay and additive disturbance, and obtained semiglobal asymptotic tracking result. Recently, in [12] (and its preliminary version in [13]), Sharma et al. presented a robust adaptive controller for the same class of systems in [6] where the robust integral of the sign of the error term in [14] was utilized in the controller design and obtained semi-global asymptotic tracking.

Review of the relevant literature highlights the fact that most of the proposed controllers for uncertain nonlinear systems with state delay fails to guarantee asymptotic stability result and additionally, almost all of the above papers (see [12] and [15]) considered the input gain matrix to be constant. Motivated by this fact, in this work we propose a continuous robust adaptive controller that can achieve asymptotic stability for a class of uncertain nonlinear systems i) subject to additive bounded input and output disturbances, ii) with a state-dependent input gain matrix, and iii) with an unknown state delay. However, since the delay value is considered to be unknown, the delay dependent terms with structured uncertainties can not be utilized in the design of the regressor matrix. In [12], Sharma et al. dealt with this challenging issue by segregating the appropriate terms when forming the linearly parameterizable function and a delayfree regressor matrix was obtained. Inspired by this, in our work, a similar segregation is utilized to obtain a delay-free regressor matrix. Also to design a robust adaptive controller that is applicable to a wider class of nonlinear systems, the robust control structure in [14] is utilized along with an adaptive component. Another novelty of our work is removing the need for the upper bound of the unstructured uncertainties by introducing a novel adaptive gain term. Lyapunov based stability analysis is provided that is fused with a LyapunovKrasovkii functional to remove time delayed terms and semiglobal asymptotic tracking is achieved.

The rest of the paper is organized in the following manner: The system model and problem statement are presented in Section II. Controller formulation and development is given in Section III while the stability analysis is presented in Section IV. Simulation studies are presented in Section V and Section VI contains some concluding remarks. 


\section{System Model \& Problem Statement}

Consider the following class of nonlinear systems ${ }^{1}$

$$
\begin{aligned}
\dot{x}_{1}= & x_{2} \\
& \vdots \\
\dot{x}_{n-1}= & x_{n} \\
\dot{x}_{n}= & f_{1}(X)+\delta_{1}(X)+f_{2}\left(X_{\tau}\right) \\
& +\delta_{2}\left(X_{\tau}\right)+b(X)\left(u+d_{1}\right)+d_{2}
\end{aligned}
$$

where $x_{i}(t) \in \mathbb{R}^{m}, i=1, \ldots, n$, are the system states, $X(t)=\left[\begin{array}{llll}x_{1}^{T} & x_{2}^{T} & \cdots & x_{n}^{T}\end{array}\right]^{T} \in \mathbb{R}^{m n}, \tau \in \mathbb{R}$ is an unknown constant arbitrarily large time delay, $f_{1}(X)$, $f_{2}\left(X_{\tau}\right) \in \mathbb{R}^{m}$ and $b(X) \in \mathbb{R}^{m \times m}$ are linearly parameterizable uncertain nonlinear functions, $\delta_{1}(X), \delta_{2}\left(X_{\tau}\right) \in \mathbb{R}^{m}$ are unknown functions, $d_{1}(t), d_{2}(t) \in \mathbb{R}^{m}$ are unknown additive nonlinear disturbances, and $u(t) \in \mathbb{R}^{m}$ is the control input. Notice that the system model in (1) can be rewritten in the following compact form

$$
M x_{1}^{(n)}=h_{1}+M f_{2 \tau}+u+d_{1}+M \delta
$$

where $M(X) \in \mathbb{R}^{m \times m}, h_{1}(X), \delta\left(X, X_{\tau}, t\right) \in \mathbb{R}^{m}$ are explicitly defined as

$$
\begin{gathered}
M \triangleq b^{-1} \quad, \quad h_{1} \triangleq M f_{1} \\
\delta \triangleq \delta_{1}(X)+\delta_{2}\left(X_{\tau}\right)+d_{2} .
\end{gathered}
$$

The system model given in (2), is assumed to satisfy the following assumptions:

Assumption 1: The nonlinear function $b$ is symmetric, positive definite, and its inverse $M$ satisfies the inequalities

$$
\underline{m}\|\xi\|^{2} \leq \xi^{T} M(\cdot) \xi \leq \bar{m}(\cdot)\|\xi\|^{2} \quad \forall \xi \in \mathbb{R}^{m}
$$

where $\bar{m}(X) \in \mathbb{R}$ is a positive, globally invertible, nondecreasing function of its argument and $\underline{m} \in \mathbb{R}$ is a positive bounding constant.

Assumption 2: The nonlinear functions, $f_{1}, f_{2}, \delta_{1}, \delta_{2}$ and $b$, are continuously differentiable up to their second derivatives (i.e., $f_{1}, f_{2}, \delta_{1}, \delta_{2}, b \in \mathcal{C}^{2}$ ).

Assumption 3: The nonlinear functions, $f_{1}, f_{2}$ and $M$, are affine in uncertain parameters.

Assumption 4: The additive disturbances, $d_{1}(t)$ and $d_{2}(t)$, are assumed to be continuously differentiable and bounded up to their second derivatives (i.e., $d_{i}(t) \in \mathcal{C}^{2}$ and $\left.d_{i}(t), \dot{d}_{i}(t), \ddot{d}_{i}(t) \in \mathcal{L}_{\infty}, i=1,2\right)$.

Our main control objective is, given a smooth desired reference trajectory $x_{r}(t) \in \mathbb{R}^{m}$ satisfying

$$
x_{r}(t) \in \mathcal{C}^{n}, x_{r}^{(i)}(t) \in \mathcal{L}_{\infty}, i=0,1, \ldots,(n+2) .
$$

ensure that the output of the system, $x_{1}(t)$, converges to the desired reference signal asymptotically. To provide a means of quantifying the control objective, we define the output tracking error signal $e_{1}(t) \in \mathbb{R}^{m}$ as

$$
e_{1} \triangleq x_{r}-x_{1} \text {. }
$$

\footnotetext{
${ }^{1}$ Throughout the paper, a time dependent delayed function is denoted as $x(t-\tau)$ or $x_{\tau}$ and a time dependent function without time delay is denoted as $x(t)$ or $x$
}

To further ease the presentation of the subsequent analysis, a combination of the reference trajectory and its time derivatives is defined as

$$
X_{r}(t)=\left[\begin{array}{llll}
x_{r}^{T} & \dot{x}_{r}^{T} & \cdots & \left(x_{r}^{(n)}\right)^{T}
\end{array}\right]^{T} \in \mathbb{R}^{m n} .
$$

In the next section, we will present the development of a full state (the system state vector $X(t)$ with all its components are measurable) robust adaptive control law that ensures $\left\|e_{1}^{(i)}(t)\right\| \rightarrow 0$ as $t \rightarrow \infty, i=0, \ldots,(n-1)$, with all signals remain bounded within the closed-loop system.

\section{Control Development}

We begin our analysis by defining, error signals, denoted by $e_{i}(t) \in \mathbb{R}^{m}, i=2,3, \ldots, n$, as follows

$$
\begin{aligned}
& e_{2} \triangleq \dot{e}_{1}+e_{1} \\
& e_{3} \triangleq \dot{e}_{2}+e_{2}+e_{1} \\
& \quad \vdots \\
& e_{n} \triangleq \dot{e}_{n-1}+e_{n-1}+e_{n-2} .
\end{aligned}
$$

A general expression for $e_{i}(t), i=2,3, \ldots, n$ in terms of $e_{1}(t)$ and its time derivatives can be obtained as

$$
e_{i}=\sum_{j=0}^{i-1} a_{i, j} e_{1}^{(j)}
$$

where the known constant coefficients $a_{i, j} \in \mathbb{R}$ are generated via a number series [14]. To facilitate the control development, filtered error signal, denoted by $r(t) \in \mathbb{R}^{m}$, is defined by

$$
r \triangleq \dot{e}_{n}+\Lambda e_{n}
$$

where $\Lambda \in \mathbb{R}^{m \times m}$ is a constant, diagonal, positive definite, gain matrix. After differentiating (13) and premultiplying the resulting equation with $M$, and applying some mathematical manipulations, the following expression can be derived

$$
M \dot{r}=-\frac{1}{2} \dot{M} r-e_{n}-\dot{u}+\tilde{N}+N_{r}+\psi
$$

where (7), (12), the first time derivative of (2), and the fact that $a_{n,(n-1)}=1$ were utilized and the auxiliary functions, $N_{r}(t), \tilde{N}(X, t), \psi(t) \in \mathbb{R}^{m}$ and $N(X, t) \in \mathbb{R}^{m}$ are explicitly defined in the following manner

$$
\begin{aligned}
N \triangleq & M\left(x_{r}^{(n+1)}+\sum_{j=0}^{n-2} a_{n j} e_{1}^{(j+2)}+\Lambda \dot{e}_{n}\right)+e_{n} \\
& +\dot{M}\left(x_{1}^{(n)}+\frac{1}{2} r\right)-\dot{h}_{1}-\dot{M} f_{2}-M \dot{f}_{2} \\
N_{r} \triangleq & \left.N\right|_{X=X_{r}, \dot{X}=\dot{X}_{r}} \\
\tilde{N} \triangleq & \left(N-\frac{d}{d t}\left(M\left(\delta+f_{2 \tau}-f_{2}\right)\right)\right) \\
& -\left(N_{r}-\frac{d}{d t}\left(M_{r}\left(\delta_{r}+f_{2 \tau r}-f_{2 r}\right)\right)\right) \\
\psi \triangleq & -\dot{d}_{1}-\frac{d}{d t}\left(M_{r}\left(\delta_{r}+f_{2 \tau r}-f_{2 r}\right)\right)
\end{aligned}
$$


in which the terms $M_{r}(t) \in \mathbb{R}^{m \times m}$ and $\delta_{r}(t), f_{2 r}(t)$, $f_{2 \tau r}(t) \in \mathbb{R}^{m}$ are given as follows

$$
\begin{aligned}
M_{r} & \left.\triangleq M\right|_{X=X_{r}} \\
\delta_{r} & \triangleq \delta_{1}\left(X_{r}(t)\right)+\delta_{2}\left(X_{r}(t-\tau)\right)+d_{2}(t) \\
f_{2 r} & =f_{2}\left(X_{r}(t)\right) \\
f_{2 \tau r} & =f_{2}\left(X_{r}(t-\tau)\right) .
\end{aligned}
$$

Note that the $N(\cdot)$ term defined in (15), does not explicitly depend on $\tau$. Also the auxiliary signals of (16), (17) and (18) are constructed such that:

Remark 1: From a direct application of the Mean Value Theorem [16] (along with Assumptions 2 and 4 ), the norm of $\tilde{N}(X, t)$ can be upper bounded in the form

$$
\|\tilde{N}(\cdot)\| \leq \rho_{1}(\|z\|)\|z\|+\rho_{2}\left(\left\|z_{\tau}\right\|\right)\left\|z_{\tau}\right\|
$$

where $\rho_{1}(\cdot), \rho_{2}(\cdot) \in \mathbb{R}$ are non-negative, globally invertible, non-decreasing functions of their arguments and $z(t) \in$ $\mathbb{R}^{(n+1) m \times 1}$ is defined by

$$
z \triangleq\left[\begin{array}{lllll}
e_{1}^{T} & e_{2}^{T} & \ldots & e_{n}^{T} & r^{T}
\end{array}\right]^{T} .
$$

Remark 2: From (6), Assumption 4, (15), (16), and (18), it can be concluded that $\psi(t), N_{r}(t), \dot{\psi}(t) \dot{N}_{r}(t) \in \mathcal{L}_{\infty}$.

Remark 3: The term $N_{r}(t)$ does not depend on the unknown time delay $\tau$, and from Assumption 3, it can be linearly parameterized in the sense that

$$
N_{r}=W_{r} \theta
$$

where $\theta \in \mathbb{R}^{p}$ is the unknown parameter vector and $W_{r}(t) \in \mathbb{R}^{m \times p}$ denotes the known regressor matrix which is a function of only $x_{r}(t)$ and its time derivatives, and does not depend on the delay.

Based on the open-loop error system in (14) and the linearly parameterized term in (25), the control input is designed as

$$
\begin{aligned}
u= & \left(K+I_{m}\right)\left[e_{n}(t)-e_{n}\left(t_{0}\right)+\Lambda \int_{t_{0}}^{t} e_{n}(\sigma) d \sigma\right] \\
& +\int_{t_{0}}^{t} W_{r}(\sigma) \hat{\theta}(\sigma) d \sigma+\Pi
\end{aligned}
$$

where the auxiliary signal $\Pi(t) \in \mathbb{R}^{m}$ is generated according to the following update law

$$
\dot{\Pi}=\left(\bar{C}_{1}+C_{2}\right) \operatorname{Sgn}\left(e_{n}\right), \Pi\left(t_{0}\right)=0_{m \times 1}
$$

and $\hat{\theta}(t) \in \mathbb{R}^{p}$ denotes the parameter estimate vector and is generated via

$$
\begin{aligned}
\hat{\theta}= & \Gamma \int_{t_{0}}^{t} W_{r}^{T}(\sigma) \Lambda e_{n}(\sigma) d \sigma-\Gamma \int_{t_{0}}^{t} \dot{W}_{r}^{T}(\sigma) e_{n}(\sigma) d \sigma \\
& +\Gamma W_{r}^{T}(t) e_{n}(t)-\Gamma W_{r}^{T}\left(t_{0}\right) e_{n}\left(t_{0}\right) .
\end{aligned}
$$

In (26)-(28), $K, C_{2} \in \mathbb{R}^{m \times m}$ and $\Gamma \in \mathbb{R}^{p \times p}$ are constant, diagonal, positive definite, gain matrices, $I_{m} \in \mathbb{R}^{m \times m}$ is the identity matrix, $\operatorname{Sgn}(\cdot) \in \mathbb{R}^{m}$ is the vector signum function, and $\bar{C}_{1}(t) \in \mathbb{R}^{m \times m}$ is a piece-wise time-varying continuous diagonal control gain matrix with its diagonal entries being defined as

$$
\bar{C}_{1 i}(t)=\left|e_{n i}(t)\right|-\left|e_{n i}\left(t_{0}\right)\right|+\Lambda_{i} \int_{t_{0}}^{t}\left|e_{n i}(\sigma)\right| d \sigma
$$

where the subscript $i=1, \cdots, m$ denotes the $i$ th element of the vector or the diagonal matrix. It should be noted that $u\left(t_{0}\right)=0_{m \times 1}, \hat{\theta}\left(t_{0}\right)=0_{p \times 1}$ and $\bar{C}_{1 i}\left(t_{0}\right)=0$ where $0_{p \times 1} \in \mathbb{R}^{p}$ and $0_{m \times 1} \in \mathbb{R}^{m}$ are vectors of zeros. Based on the structure of (26)-(29), the following are obtained

$$
\begin{aligned}
\dot{u} & =\left(K+I_{m}\right) r+W_{r} \hat{\theta}+\left(\bar{C}_{1}+C_{2}\right) \operatorname{Sgn}\left(e_{n}\right)(30) \\
\dot{\hat{\theta}} & =\Gamma W_{r}^{T} r \\
\dot{\bar{C}}_{1 i} & =r_{i} \operatorname{sgn}\left(e_{n i}\right), i=1, \cdots, m
\end{aligned}
$$

where (13) was utilized. At this stage, substituting (30) into (14), the following closed-loop error system for $r(t)$ is obtained

$$
\begin{aligned}
M \dot{r}= & -\frac{1}{2} \dot{M} r-e_{n}-\left(K+I_{m}\right) r+W_{r} \tilde{\theta}- \\
& \left(\bar{C}_{1}+C_{2}\right) \operatorname{Sgn}\left(e_{n}\right)+\tilde{N}+\psi
\end{aligned}
$$

where the parameter estimation error signal $\tilde{\theta}(t) \in \mathbb{R}^{p}$ is defined as

$$
\tilde{\theta} \triangleq \theta-\hat{\theta} .
$$

\section{Stability ANalysis}

Before introducing the stability result, we would like to present two preliminary lemmas, where the output of both will later be utilized in the proof of our main result.

Lemma 1: Let the auxiliary function $L_{1}(t) \in \mathbb{R}$ be defined as follows

$$
L_{1} \triangleq r^{T}\left(\psi-C_{1} \operatorname{Sgn}\left(e_{n}\right)\right)
$$

where $C_{1} \in \mathbb{R}^{m \times m}$ is a constant, diagonal, positive definite, bounding matrix. If the entries of $C_{1}$ are selected to satisfy the following sufficient condition

$$
C_{1 i}>\left\|\psi_{i}(t)\right\|_{\mathcal{L}_{\infty}}+\frac{1}{\Lambda_{i}}\left\|\dot{\psi}_{i}(t)\right\|_{\mathcal{L}_{\infty}}
$$

then the following inequality is satisfied

$$
\int_{t_{0}}^{t} L_{1}(\tau) d \tau \leq \zeta_{b 1}
$$

where $\zeta_{b 1} \in \mathbb{R}$ is a positive bounding constant.

Proof: A similar proof can be found in [14].

Remark 4: It is highlighted that the bounding matrix in (35) (i.e., $C_{1}$ ) was not utilized in the control design (see (26)-(28)). Instead, the update rule given in (29) was utilized in the controller. This is a novel departure from the similar existing results in the literature. Specifically, in [12] and [13], Sharma et al. used a similar term to that of (27) but with a constant control parameter (which should be greater than the uncertainty bounds). In this study, different from [12] and [13], the term in (27) is designed with a piece-wise time-varying continuous control gain matrix which does not require the knowledge of the uncertainty bounds. 
Lemma 2: The auxiliary function $L_{2}(t) \in \mathbb{R}$ is defined as

$$
L_{2} \triangleq-C_{2} \dot{e}_{n} \operatorname{sgn}\left(e_{n}\right) \text {. }
$$

Provided that $C_{2}$ is positive definite then

$$
\int_{t_{0}}^{t} L_{2}(\sigma) d \sigma \leq \zeta_{b_{2}}
$$

where $\zeta_{b_{2}} \in \mathbb{R}$ is a positive constant.

Proof: See Appendix I.

We are ready to propose the following Theorem:

Theorem 1: The control law (26), (27), with the update law (28), and the time-varying gain introduced in (29) ensure the boundedness of all closed-loop system signals and the convergence of the error signals in the sense that

$$
\lim _{t \rightarrow \infty}\left\|e_{1}^{(i)}(t)\right\|=0 \text { for } i=0, \ldots, n
$$

provided that

$$
\lambda_{\min }(\Lambda)>\frac{1}{2}
$$

and the entries of the controller gain matrix $K$ defined in (26) selected sufficiently large relative to the system initial conditions.

Proof: We begin our proof by defining the following non-negative scalar function, denoted by $V(s(t), t) \in \mathbb{R}$, as

$$
\begin{aligned}
V \triangleq & \frac{1}{2} \sum_{i=1}^{n} e_{i}^{T} e_{i}+\frac{1}{2} r^{T} M r+\frac{1}{2} \tilde{\theta}^{T} \Gamma^{-1} \tilde{\theta} \\
& +P_{1}+P_{2}+P_{3}+P_{4}
\end{aligned}
$$

where $P_{1}(t), P_{2}(t), P_{3}(t), P_{4}(t) \in \mathbb{R}$ are explicitly defined in the following manner

$$
\begin{gathered}
P_{1} \triangleq \zeta_{b 1}-\int_{t_{0}}^{t} L_{1}(\sigma) d \sigma \\
P_{2} \triangleq \frac{1}{2 K_{\min }} \int_{t-\tau}^{t} \rho_{2}^{2}(\|z(\sigma)\|)\|z(\sigma)\|^{2} d \sigma \\
P_{3} \triangleq \zeta_{b 2}-\int_{t_{0}}^{t} L_{2}(\sigma) d \sigma \\
P_{4} \triangleq \frac{1}{2} \sum_{i=1}^{m}\left(C_{1 i}-\bar{C}_{1 i}\right)^{2}
\end{gathered}
$$

with $K_{\min } \in \mathbb{R}$ being the minimum eigenvalue of $K$ and $s(t) \in \mathbb{R}^{[(n+1) m+4+p] \times 1}$ is defined as

$$
s=\left[\begin{array}{llllll}
z^{T} & \sqrt{P_{1}} & \tilde{\theta}^{T} & \sqrt{P_{2}} & \sqrt{P_{3}} & \sqrt{P_{4}}
\end{array}\right]^{T} .
$$

Note that, after utilizing (5), (42) can be bounded in the following way

$$
\lambda_{1}\|s\|^{2} \leq V(s, t) \leq \lambda_{2}(\|s\|)\|s\|^{2}
$$

where $\lambda_{1}, \lambda_{2}(\cdot) \in \mathbb{R}$ are defined as

$$
\begin{aligned}
& \lambda_{1}=\frac{1}{2} \min \left\{1, \underline{m}, \lambda_{\min }\left(\Gamma^{-1}\right)\right\} \\
& \lambda_{2}=\max \left\{1, \frac{1}{2} \bar{m}(\|s\|), \frac{1}{2} \lambda_{\max }\left(\Gamma^{-1}\right)\right\} .
\end{aligned}
$$

Taking the time derivative of (42), utilizing

$$
\begin{aligned}
\sum_{i=1}^{n} e_{i}^{T} \dot{e}_{i}= & e_{1}^{T}\left(e_{2}-e_{1}\right)+e_{2}^{T}\left(e_{3}-e_{2}-e_{1}\right)+\ldots \\
& +e_{n-1}^{T}\left(e_{n}-e_{n-1}-e_{n-2}\right)+e_{n}^{T}\left(r-\Lambda e_{n}\right) \\
= & -\sum_{i=1}^{n-1} e_{i}^{T} e_{i}+e_{n-1}^{T} e_{n}+e_{n}^{T} r-e_{n}^{T} \Lambda e_{n}
\end{aligned}
$$

and substituting for (31), (33), (35), (38) then cancelling the cross terms, we obtain

$$
\begin{aligned}
\dot{V}= & -\sum_{i=1}^{n-1} e_{i}^{T} e_{i}-e_{n}^{T} \Lambda e_{n}+e_{n-1}^{T} e_{n}-r^{T} r \\
& +r^{T} \tilde{N}-r^{T} K r+\frac{1}{2 K_{\min }} \rho_{2}^{2}(\|z\|)\|z\|^{2} \\
& -\frac{1}{2 K_{\min }} \rho_{2}^{2}\left(\left\|z_{\tau}\right\|\right)\left\|z_{\tau}\right\|^{2}-e_{n}^{T} \Lambda C_{2} \operatorname{Sgn}\left(e_{n}\right)(51)
\end{aligned}
$$

which can be upper bounded in the following way

$$
\begin{aligned}
\dot{V} \leq & -\lambda_{3}\|z\|^{2}+\|r\| \rho_{1}(\|z\|)\|z\|+\|r\| \rho_{2}\left(\left\|z_{\tau}\right\|\right)\left\|z_{\tau}\right\| \\
& -K_{\min }\|r\|^{2}+\frac{1}{2 K_{\min }} \rho_{2}^{2}(\|z\|)\|z\|^{2} \\
& -\frac{1}{2 K_{\min }} \rho_{2}^{2}\left(\left\|z_{\tau}\right\|\right)\left\|z_{\tau}\right\|^{2}-\sum_{i=1}^{m} \Lambda_{i} C_{2 i}\left|e_{n i}\right| .
\end{aligned}
$$

Utilizing

$$
\begin{gathered}
\|r\| \rho_{2}\left(\left\|z_{\tau}\right\|\right)\left\|z_{\tau}\right\| \leq \frac{K_{\min }}{2}\|r\|^{2}+\frac{1}{2 K_{\min }} \rho_{2}^{2}\left(\left\|z_{\tau}\right\|\right)\left\|z_{\tau}\right\|^{2} \\
\|r\| \rho_{1}(\|z\|)\|z\| \leq \frac{K_{\min }}{2}\|r\|^{2}+\frac{1}{2 K_{\min }} \rho_{1}^{2}(\|z\|)\|z\|^{2}
\end{gathered}
$$

we can further upper bound (52) as

$$
\dot{V} \leq-\left(\lambda_{3}-\frac{\rho(\|z\|)}{2 K_{\min }}\right)\|z\|^{2}-\sum_{i=1}^{m} \Lambda_{i} C_{2 i}\left|e_{n i}\right|
$$

where $\lambda_{3} \triangleq \min \left\{\frac{1}{2}, \lambda_{\min }(\Lambda)-\frac{1}{2}\right\}$ and $\rho(\|z\|) \triangleq$ $\rho_{1}^{2}(\|z\|)+\rho_{2}^{2}(\|z\|)$.

Provided that the minimum eigenvalue of the controller gain, $K_{\min }$, is selected according to satisfy

$$
K_{\min } \geq \frac{\rho(\|z\|)}{2 \lambda_{3}} \text { or }\|z\| \leq \rho^{-1}\left(2 \lambda_{3} K_{\min }\right)
$$

the upper bound of $\dot{V}(t)$ given in (55) can be formulated to have the following form

$$
\dot{V} \leq-\beta_{0}\|z\|^{2}-\sum_{i=1}^{m} \Lambda_{i} C_{2 i}\left|e_{n i}\right|
$$

where $\beta_{0} \in \mathbb{R}$ is a positive constant. From the structure of (42) and (57), we can conclude that $V(s, t) \in \mathcal{L}_{\infty}$ when (56) is satisfied. And since $V(s, t)$ is a decreasing function it should have its maximum value at $V\left(s\left(t_{0}\right), t_{0}\right)$. This enables us to further update the gain condition to have the following form

$$
K_{\min } \geq \frac{1}{2 \lambda_{3}} \rho\left(\sqrt{\frac{\lambda_{2}\left(\left\|s\left(t_{0}\right)\right\|\right)}{\lambda_{1}}}\left\|s\left(t_{0}\right)\right\|\right) .
$$


Following standard signal chasing methodologies we can prove that all signals in the closed-loop system is bounded when the gain condition (58) is satisfied. Furthermore from the integral of (57) we can prove that $z(t) \in \mathcal{L}_{2}$ and $e_{n}(t) \in$ $\mathcal{L}_{1}$. Since $e_{n}(t) \in \mathcal{L}_{1} \cap \mathcal{L}_{\infty}$, from (29), we can conclude that $\bar{C}_{1}(t) \in \mathcal{L}_{\infty}$, and since $r(t) \in \mathcal{L}_{\infty}$, then from (32), it is clear that $\dot{\bar{C}}_{1}(t) \in \mathcal{L}_{\infty}$. A direct application of Theorem 8.4 in [16] can be used to prove that $\|z(t)\| \rightarrow 0$ when the gain condition (58) is satisfied. Based on the definition of $z(t)$, it is easy to show that $\left\|e_{i}(t)\right\|,\|r(t)\| \rightarrow 0$ as $t \rightarrow \infty$, $i=1,2, \ldots, n$. From (13), it is clear that $\left\|\dot{e}_{n}(t)\right\| \rightarrow 0$ as $t \rightarrow \infty$. By utilizing (12) recursively it can be proven that $\left\|e_{1}^{(i)}(t)\right\| \rightarrow 0$ as $t \rightarrow \infty, i=1,2, \ldots, n$.

Notice that, from (58), it is apparent that our proof depends on the selection of the controller gain according to the initial conditions of the system. Therefore the stability result is semi-global.

\section{Numerical Simulation Results}

A numerical simulation was performed to demonstrate the performance of the adaptive controller given in (26)-(29). The class of systems in (1) with $m=n=2$ is considered with the following modeling functions

$$
\begin{aligned}
f_{1} & =\left[\phi_{3} x_{21} x_{12}, \phi_{4} x_{11} x_{22}\right]^{T} \\
f_{2} & =\left[\phi_{5} x_{11 \tau} x_{22 \tau}, \phi_{6} \cos \left(x_{12 \tau}\right)\right]^{T} \\
b & =\operatorname{diag}\left\{\frac{2+\cos x_{11}}{\phi_{1}}, \frac{3+\sin x_{12}}{\phi_{2}}\right\} \\
\delta_{1} & =\left[\sin \left(5 x_{21}\right), \cos \left(3 x_{12}\right)\right]^{T} \\
\delta_{2} & =\left[0.5\left(x_{11 \tau} x_{21 \tau}+x_{12 \tau} x_{22 \tau}\right), \sin \left(2 x_{11 \tau}\right)\right]^{T} \\
d_{1} & =[\cos (2 t)+\exp (-0.5 t), \sin (3 t)+\exp (-0.5 t)]^{T} \\
d_{2} & =[\sin (2 t)+\exp (-0.5 t), \cos (3 t)+\exp (-0.5 t)]^{T}
\end{aligned}
$$

with $x_{1}=\left[\begin{array}{ll}x_{11} & x_{12}\end{array}\right]^{T}$ and $x_{2}=\left[\begin{array}{ll}x_{21} & x_{22}\end{array}\right]^{T}$. The reference trajectory was selected as

$$
x_{r}=\left[\begin{array}{c}
\sin (t)\left(1-\exp \left(-0.2 t^{3}\right)\right) \\
\cos (t)\left(1-\exp \left(-0.5 t^{3}\right)\right)
\end{array}\right] .
$$

During simulation studies the initial conditions were set to $x_{1}\left(t_{0}\right)=\left[\begin{array}{ll}-1 & -1\end{array}\right]^{T}, x_{2}\left(t_{0}\right)=\left[\begin{array}{ll}0 & 0\end{array}\right]^{T}, \hat{\theta}\left(t_{0}\right)=$ $[0.75,0.9,0.5,0.6,0.9,0.8]^{T}$, while the controller gains were chosen via trial and error method as $\Lambda=5 I_{2}, K=10 I_{2}$, $C_{2}=3 I_{2}, \Gamma=5 I_{6}$, and $\tau=10 \mathrm{sec}$. The unknown parameter vector is formed as $\theta=\left[\phi_{1}, \phi_{2}, \phi_{1} \phi_{3}, \phi_{1} \phi_{5}, \phi_{2} \phi_{4}, \phi_{2} \phi_{6}\right]^{T}$ where unknown parameters are given as $\phi_{1}=0.5, \phi_{2}=0.2$, $\phi_{3}=\phi_{6}=2, \phi_{4}=\phi_{5}=3$. The tracking error $e_{1}(t)$ is presented in Figure 1. In Figures 2 and 3, the control input $u(t)$ and the parameter estimate $\hat{\theta}(t)$ are presented, respectively. Finally, the entries of the time-varying gain matrix $\bar{C}_{1}(t)$ are presented in Figure 4.

\section{CONCLUSION}

In this work, we have developed a singularity-free robust adaptive controller for a class of MIMO nonlinear systems with unknown state delay which is subject to both parametric and non-parameterizable uncertainties along with additive input and output disturbances. An adaptive desired system compensation in conjunction with a continuous nonlinear integral feedback component is utilized in the design of the controller and Lyapunov-based techniques, are used to prove that the tracking error is asymptotically driven to zero. Our stability analysis parallels the previous result presented in [12] however the proposed methodology can be applied to a wider class of nonlinear systems. Additionally, by introducing a time-varying gain (i.e., $\bar{C}_{1}(t)$ ), the need for knowledge of the upper bounds of the unstructured uncertainties was removed which is a novel departure from the previous results including [12]. When compared with the previous robust adaptive type of control for time delay systems, we considered a state-dependent input gain matrix for the system model and the our controller controller is robust to both matched and unmatched disturbances. A numerical simulation was presented to demonstrate the viability and performance of the proposed method.
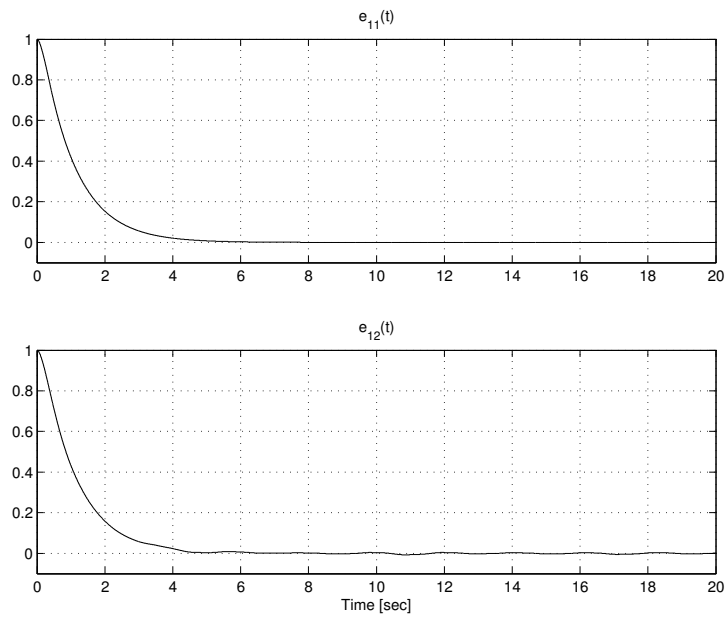

Fig. 1. Tracking Error $e_{1}(t)$
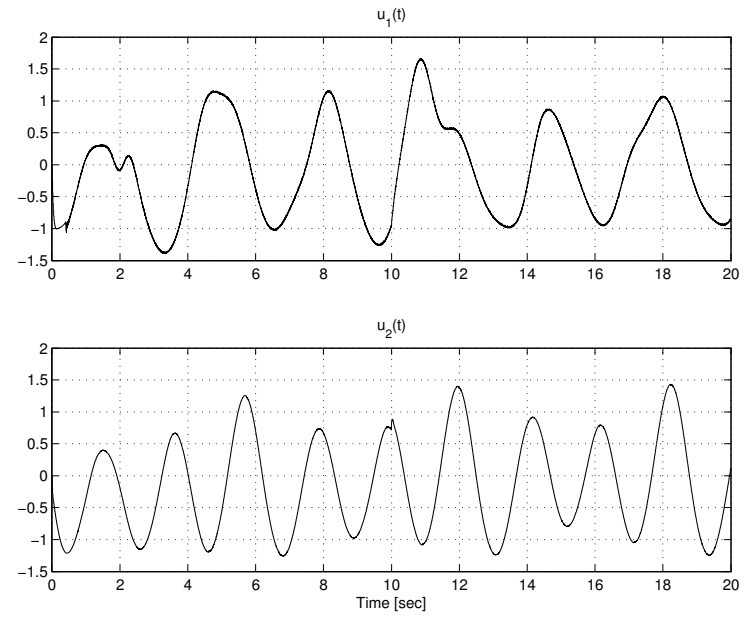

Fig. 2. Control Input $u(t)$ 


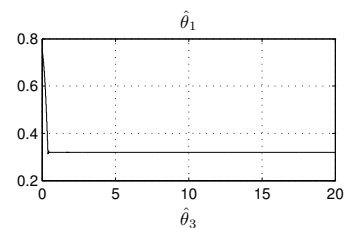

$\hat{\theta}_{3}$
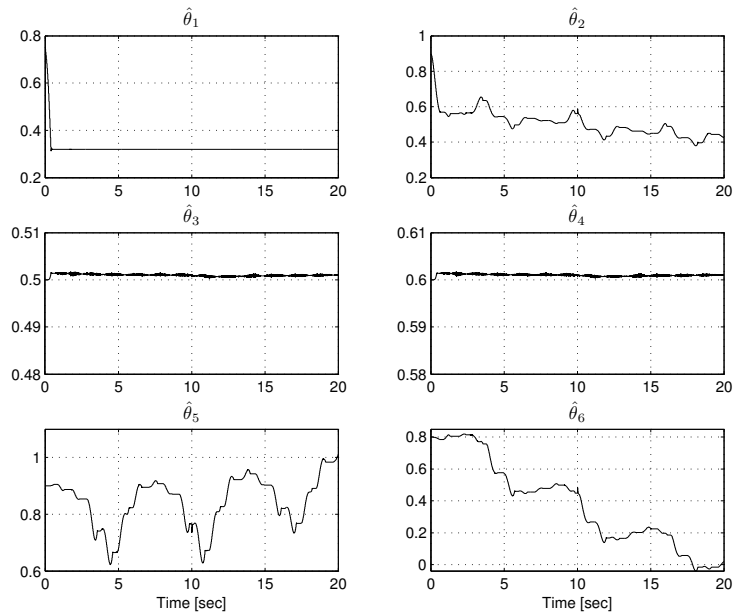

Fig. 3. Parameter Estimate $\hat{\theta}(t)$
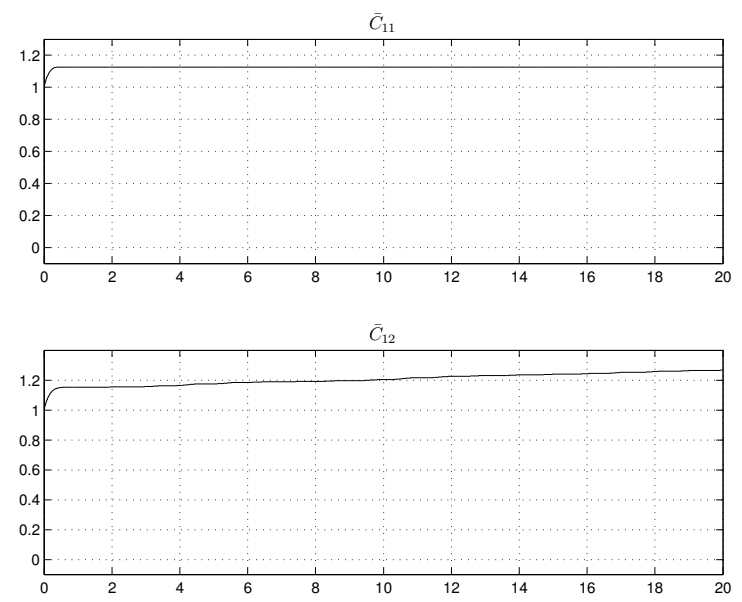

Fig. 4. Diagonal entries of the time-varying control gain matrix $\bar{C}_{1}(t)$

\section{APPENDIX I \\ PROOF OF LEMMA 2}

Proof: The integral of (38) from $t_{0}$ to $t$ is obtained as follows [17]

$$
\begin{aligned}
\int_{t_{0}}^{t} L_{2}(\tau) d \tau & =-C_{2} \int_{t_{0}}^{t} \dot{e}_{n}(\tau) \operatorname{Sgn}\left(e_{n}(\tau)\right) d \tau \\
& =-C_{2} \int_{t_{0}}^{t} \operatorname{Sgn}\left(e_{n}\right) d\left(e_{n}\right) \\
& =-C_{2} \int_{t_{0}}^{t} d\left(\left|e_{n}\right|\right) \\
& =-C_{2}\left(\left|e_{n}(t)\right|-\left|e_{n}\left(t_{0}\right)\right|\right) \\
& \leq C_{2}\left|e_{n}\left(t_{0}\right)\right|
\end{aligned}
$$

It can be easily seen that if $C_{2}$ is chosen as positive, then the inequality in (39) holds with

$$
\xi_{b 2} \triangleq C_{2}\left|e_{n}\left(t_{0}\right)\right| \text {. }
$$

\section{REFERENCES}

[1] J.-P. Richard, "Time-delay systems: An overview of some recent advances and open problems," Automatica, vol. 39, no. 10, pp. 1667$1694,2003$.

[2] T. P. Zhang and S. S. Ge, "Adaptive neural control of MIMO nonlinear state time-varying delay systems with unknown dead-zones and gain signs," Automatica, vol. 43, no. 6, pp. 1021-1033, 2007.

[3] B. Chen, X. Liu, K. Liu, and C. Lin, "Novel adaptive neural control design for nonlinear MIMO time-delay systems," Automatica, vol. 45, no. 6, pp. 1554-1560, 2009.

[4] S. Ge, F. Hong, and T. H. Lee, "Adaptive neural network control of nonlinear systems with unknown time delays," IEEE Trans. Autom. Control, vol. 48, no. 11, pp. 2004-2010, 2003.

[5] D. W. C. Ho, J. Li, and Y. Niu, "Adaptive neural control for a class of nonlinearly parametric time-delay systems," IEEE Trans. Neural Netw., vol. 16, no. 3, pp. 625-635, 2005.

[6] S. Ge, F. Hong, and T. H. Lee, "Robust adaptive control of nonlinear systems with unknown time delays," Automatica, vol. 41, no. 7, pp 1181-1190, 2005.

[7] F. Zheng, Q.-G. Wang, and T. H. Lee, "Adaptive robust control of uncertain time delay systems," Automatica, vol. 41, no. 8, pp. 1375$1383,2005$.

[8] C. Hua, G. Feng, and X. Guan, "Robust controller design of a class of nonlinear time delay systems via backstepping method," Automatica, vol. 44, no. 2, pp. 567-573, 2008.

[9] S.-C. Tong and N. Sheng, "Adaptive fuzzy observer backstepping control for a class of uncertain nonlinear systems with unknown timedelay," Int. J. of Automation and Computing, vol. 7, no. 2, pp. 236-246, 2010.

[10] $\mathrm{H}$. Wu, "Adaptive robust control of uncertain nonlinear systems with nonlinear delayed state perturbations," Automatica, vol. 45, no. 8, pp. 1979-1984, 2009.

[11] B. Mirkin and P.-O. Gutman, "Adaptive output-feedback tracking: The case of MIMO plants with unknown, time-varying state delay," Systems \& Control Letters, vol. 58, no. 1, pp. 62-68, 2009.

[12] N. Sharma, S. Bhasin, Q. Wang, and W. E. Dixon, "RISE-based adaptive control of a control affine uncertain nonlinear system with unknown state delays," IEEE Trans. Autom. Control, vol. 57, no. 1, pp. 255-259, 2012.

[13] - ,RISE-based adaptive control of an uncertain nonlinear system with unknown state delays," in Proc. IEEE Int. Conf. Decision and Control, Atlanta, GA, USA, 2010, pp. 1773-1778.

[14] B. Xian, D. M. Dawson, M. S. de Queiroz, and J. Chen, "A continuous asymptotic tracking control strategy for uncertain nonlinear systems," IEEE Trans. Autom. Control, vol. 49, no. 7, pp. 1206-1211, 2004

[15] B. Mirkin, P. Gutman, and Y. Shtessel, "Continuous model reference adaptive control with sliding mode for a class of nonlinear plants with unknown state delay," in Proc. American Control Conf., St. Louis, MO, USA, 2009, pp. 574-579.

[16] H. K. Khalil, Nonlinear Systems, 3rd Edition. New York, NY, USA Prentice Hall, 2002.

[17] M. Krstic, Delay Compensation for Nonlinear, Adaptive, and PDE Systems. Boston, MA, USA: Birkhauser, 2009. 